\title{
Strain rate effect on the mechanical behavior of the anterior cruciate ligament-bone complex
}

\author{
D.P. Pioletti ${ }^{\mathrm{a}, \mathrm{b}, *}$, L.R. Rakotomanana ${ }^{\mathrm{a}, \mathrm{b}}$, P.-F. Leyvraz ${ }^{\mathrm{a}}$ \\ a Hopital Orthopédique de la Suisse Romande, Lausanne, Switzerland \\ ${ }^{\mathrm{b}}$ Biomedical Engineering Laboratory, Swiss Federal Institute of Technology, Lausanne, Switzerland
}

Received 2 October 1998; received in revised form 26 January 1999; accepted 22 February 1999

\begin{abstract}
Traction tests were performed on the bovine anterior cruciate ligament-bone complex at seven strain rates $(0.1,1,5,10,20,30$, $40 \% / \mathrm{s})$. Corresponding stress-strain curves showed that, for a given strain level, the stress increased with the augmentation of the strain rate. This phenomenon was important since the stress increased by a factor of three between the tests performed at the lowest and highest strain rates. The influence of the strain rate was quantified with a new variable called the "supplemental stress". This variable represented the percentage of total stress due to the effect of strain rate. It was observed that at a strain rate of $40 \% / \mathrm{s}$, more than $70 \%$ of the stress in the ligament was due to the strain rate effect. In fact, the strain rate strongly affected the toe region, but did not influence the linear part of the stress-strain curves. The use of the linear tangent moduli was then not adequate to describe the strain rate effect in the anterior cruciate ligament-bone complex. This study showed that the "supplemental stress" was a synthetic and convenient variable to quantify the effect of the strain rate on the entire stress-strain curves. This quantification is especially important when comparing the mechanical behavior between anterior cruciate ligament and tissues used as ligament graft. (C) 1999 IPEM. Published by Elsevier Science Ltd. All rights reserved.
\end{abstract}

Keywords: Stress-strain curves; Quantification of the strain rate effect; Supplemental stress

\section{Introduction}

Rupture of the anterior cruciate ligament (ACL) is a frequent clinical problem, especially in the young active population [1]. If the knee is not treated to restore its initial stability, degenerative changes in the articular cartilage are possible [2]. The surgical treatment of an ACL deficient knee has then become a routine procedure; despite its frequency, however, its success is inconsistent [3].

Consequently, numerous experimental studies have been performed to characterize the mechanical behavior of the ACL. These studies generally consist of traction tests performed at one strain rate [4-7]. From these traction tests, the stress-strain curve can be obtained, and the mechanical characterization of the ligaments is often

* Corresponding author at: Department of Bioengineering, University of California, San Diego 9500, Gilman Drive, La Jolla, CA 920930412, USA. Tel.: + 1-619-534-4272; fax: + 1-619-534-6896; e-mail: dpiolett@bioeng.ucsd.edu achieved by determination of the linear tangent modulus [8]. This quantity allows the comparison of traction tests between studies and specimens.

The knee works under dynamical loadings. Accordingly, traction tests on the ACL should be performed at different strain rates. More precisely, the dependence of the stress on the strain rate should be quantified. As noted by Danto and Woo [9], relatively few studies have evaluated the effect of strain rate on the mechanical properties of the ACL. Moreover, when traction tests are performed at different strain rates, the resulting stress strain curves are usually presented without a specific method to quantify the strain rate effect.

Woo and Blomstrom [10] have proposed that "ideally, a replacement graft should possess the same properties as the natural ACL". When comparing mechanical behavior between ACL and replacement grafts, it is then important to consider the entire stress-strain curve. This is also true for traction tests performed at different strain rates. The quantification of the strain rate effect should not be limited to the comparison of some parameters 
between different tissues, but should be performed on the entire stress-strain curves.

In this study, we present a new variable, the "supplemental stress", that describes the effect of the strain rate on the entire stress-strain curves. The results of the traction tests performed on ACL at different strain rates will be analyzed with this new variable. Moreover, we will test the hypothesis that the strain rate effect acts mainly in the toe region with minimal influence on the linear part of the stress-strain curves.

\section{Material and methods}

\subsection{Experimental set-up}

A custom made device was developed to perform uniaxial dynamical tests on isolated ligaments (Fig. 1) [11]. The forces acting on the ligament were measured by means of three Piezo Transducers (KISTLER, 9211, Winterthur, Switzerland) placed at the fixed end of the ligament (resolution: $0.24 \mathrm{~N}$ ). The strain was measured with a Linear Displacement Transducer (VIBROMETER, WG 173, Fribourg, Switzerland) placed on the moving end of the ligament (resolution: $6 \mu \mathrm{m}$ ). Special clamps were designed to prevent any slippage between the specimen and the clamps (Fig. 2). Data acquisition was performed on a Macintosh computer (Apple Computer, Cupertino, USA) through an acquisition card MACADIOS II Jr. (GW Instruments, Somerville, USA) and the software LABVIEW (National Instrument Corporation, Austin, USA). The software allowed real-time

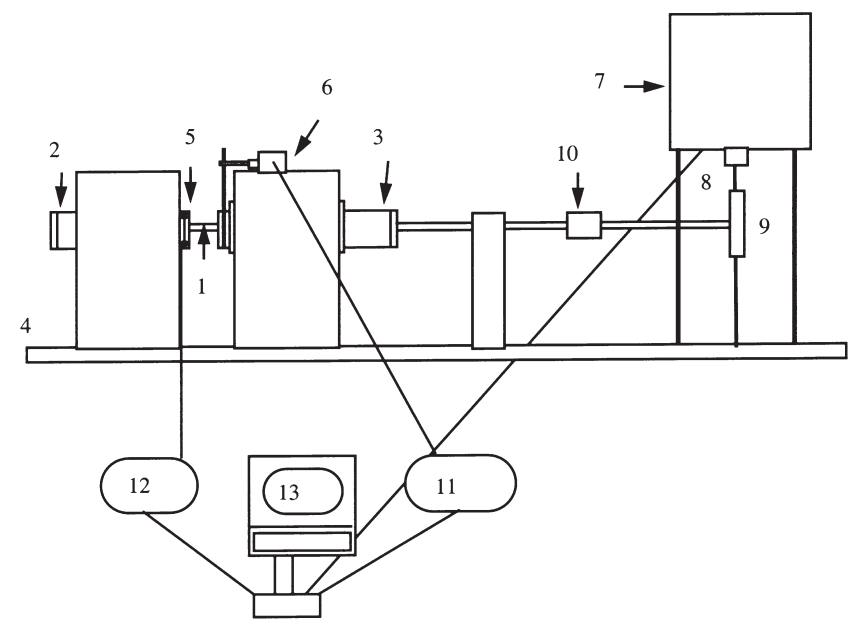

Fig. 1. View of the experimental custom-made device. The rotational motion of a D.C. motor (7) was transformed to a linear one through an high precision ball screw (10). Decoupling was possible with an endless screw (8) and a toothed wheel (9). The ligament (1) was placed in a mobile (3) and fixed (2) supports. Forces and displacements were measured with electronic transducers $(5,6)$. These measures through their specific signal processing $(12,11)$ were acquired on a personal computer with the software LabVIEW (13). control by using correctly compiled external routines written in high level language. The acquisition frequency of the system was $8 \mathrm{kHz}$.

\subsection{Specimen description}

Eleven ACL from 4-month-old bovine calves were obtained $24 \mathrm{~h}$ after death and frozen at $-24^{\circ} \mathrm{C}$, which does not influence the mechanical behavior of the ligaments [12]. From dissection to experimentation, an identical protocol was followed for all specimens. The ligaments were isolated from the joints keeping only the bone insertions. These insertions were potted in a synthetic resin (monomer beracryl). The solidification process took place in a freezer in order to minimize the heat effects on the ligaments. Experiments were performed at room temperature $\left(21^{\circ} \mathrm{C}\right)$, and the specimens were continuously kept moist using a drip with a physiological fluid $(1 / 3 \mathrm{NaCl} 0.9 \%, 2 / 3$ glucose $5 \%)$.

\subsection{Dimension measurements}

The length and cross-sectional area of the ligaments were measured when the specimens were positioned on the set-up with a $2 \mathrm{~N}$ force applied (reference configuration). The length was defined as the distance between the two bone insertions and was measured with a caliper square (resolution: $0.5 \mathrm{~mm}$ ). Cross-sectional area was assumed to be elliptic and the two principal diameters were measured in the middle of the ligaments with a caliper square. When compared to an optical device (resolution $10 \mu \mathrm{m}$ ) developed for a previous study [13], differences in diameter measurements were less than $0.5 \mathrm{~mm}$. Mean dimensions of the 11 specimens are reported in Table 1.

\subsection{Mechanical tests}

A slight rotation perpendicular to the longitudinal axis of the ligament was imposed on the specimens placed in the set-up to maximize the alignment of fibers in the traction direction. Thirty minutes before the first test, the ligaments were preconditioned with a traction performed at the lowest strain rate until $300 \mathrm{~N}$. This force corresponded for all the specimens to a strain lower than $10 \%$. This strain limit was chosen to avoid plastic deformation or damage to the specimen which would render later comparisons inappropriate [14]. To minimize the effect of the strain history, an interval of $30 \mathrm{~min}$ between each test was adopted. This time scale corresponded to that found in the literature [15]. Each ligament was then loaded until $300 \mathrm{~N}$ with seven different strain rates, always in the following order: $0.1,1,5,10,20,30$ and $40 \% / \mathrm{s}$. To check that the order of the tests did not affect the results, at the end of the tests the specimens were reloaded with the 0.1 and 5\%/s strain rates. When plotted 


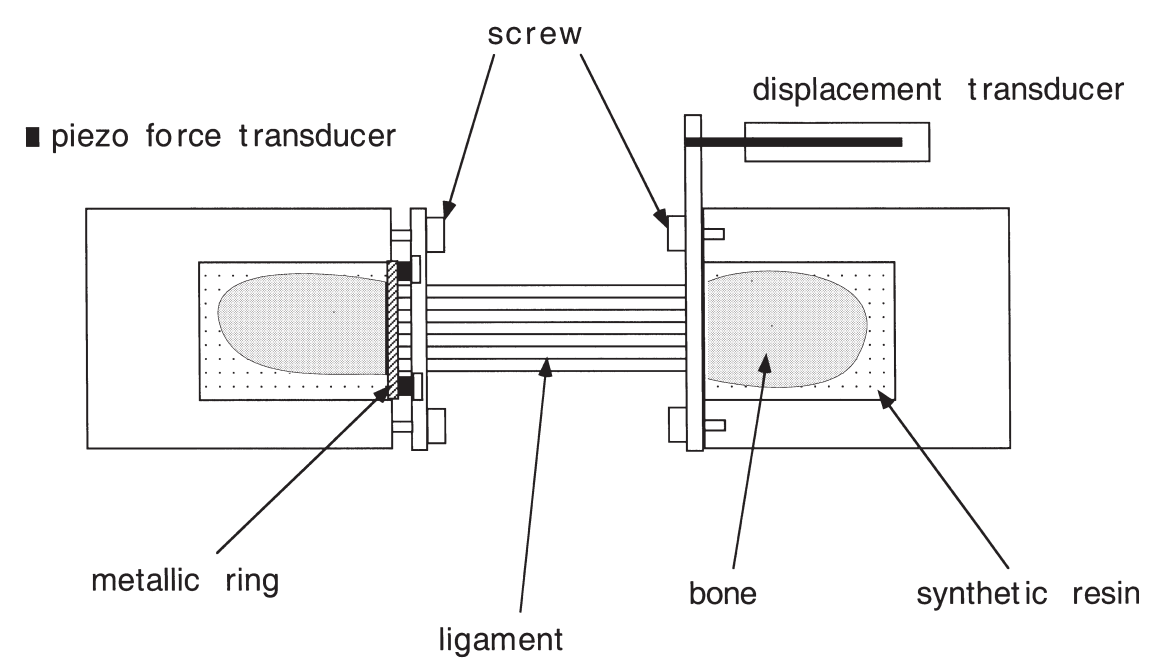

Fig. 2. View of the clamps used. The bone insertions of the ligament were potted in a synthetic resin and were clamped in metallic supports. The forces was directly measured on the ligament. To optimize the contact surfaces for the force measurements, a metallic ring was placed between the synthetic resin and the piezo force transducers.

Table 1

Mean dimensions and standard deviations of the 11 ACL specimens used

\begin{tabular}{llll}
\hline $\begin{array}{l}\text { Length } \\
(\mathrm{mm})\end{array}$ & $\begin{array}{l}\text { Cross-sectional area } \\
\left(\mathrm{mm}^{2}\right)\end{array}$ & $\begin{array}{l}\text { Major diameter } \\
(\mathrm{mm})\end{array}$ & $\begin{array}{l}\text { Minor diameter } \\
(\mathrm{mm})\end{array}$ \\
\hline $33.1 \pm 2.9$ & $91.4 \pm 12.5$ & $11.4 \pm 1.4$ & $10.1 \pm 1.5$ \\
\hline
\end{tabular}

together, the initial and reloaded stress-strain curves were identical. Complete tests were also repeated for four specimens with a different rotation value without showing difference in the stress-strain curves obtained.

\subsection{Variables definition}

The stress was defined as the actual force divided by the reference cross-sectional area. The strain was defined as the change of length divided by the reference length. The linear tangent modulus was given by the slope of the linear part of the stress-strain curves and was measured at the final third of the curves. A linear regression fit was performed in the linear part of the stress-strain curve to determine its slope value.

In order to quantify the strain rate effect on the stressstrain curves, we defined the "supplemental stress" variable as the ratio:

$$
\begin{aligned}
& \text { Supplemental stress }= \\
& \frac{\text { Stress (at } y \% / \mathrm{s} \text { strain rate) }- \text { stress (at } 0.1 \% / \mathrm{s} \text { strain rate) }}{\text { Stress (at } y \% / \mathrm{s} \text { strain rate) }} \\
& \times 100
\end{aligned}
$$

where $y$ represented the different strain rate values (1, $5,10,20,30$ and 40\%/s). The "supplemental stress" rep- resented the percentage of total stress due to the effect of strain rate. The "supplemental stress" was reported for different values of strains and strain rates.

\subsection{Statistical analysis}

A one-way ANOVA test was used to analyze the mean variance of the linear tangent moduli and of the "supplemental stress" obtained at different strain rates. If statistically significant differences were found, a least significant difference (Fisher test) was performed to determine which means were different from which other means [16]. A 95\% confidence level was selected to define significance for all statistical tests.

\section{Results}

The stress-strain curves obtained at seven strain rates presented similar trends for all the specimens. The strain rate effect was clearly visible (Fig. 3). At $4 \%$ strain, the corresponding stress increased by a factor of three between the lowest and highest strain rates. For all of the specimens, the differences between stress-strain curves obtained at different strain rates were mainly observed in the beginning of the curves (toe region). The second part of the curves, which was almost linear, was not much affected by the increase of strain rate. This observation was confirmed by the measure of the linear tangent moduli. Mean values of the linear tangent moduli obtained at seven strain rates are reported in Table 2. No statistical difference was observed between the means of the linear tangent moduli. Therefore, the linear tangent moduli are not adequate to characterize the strain rate effect of the ACL-bone complex. 


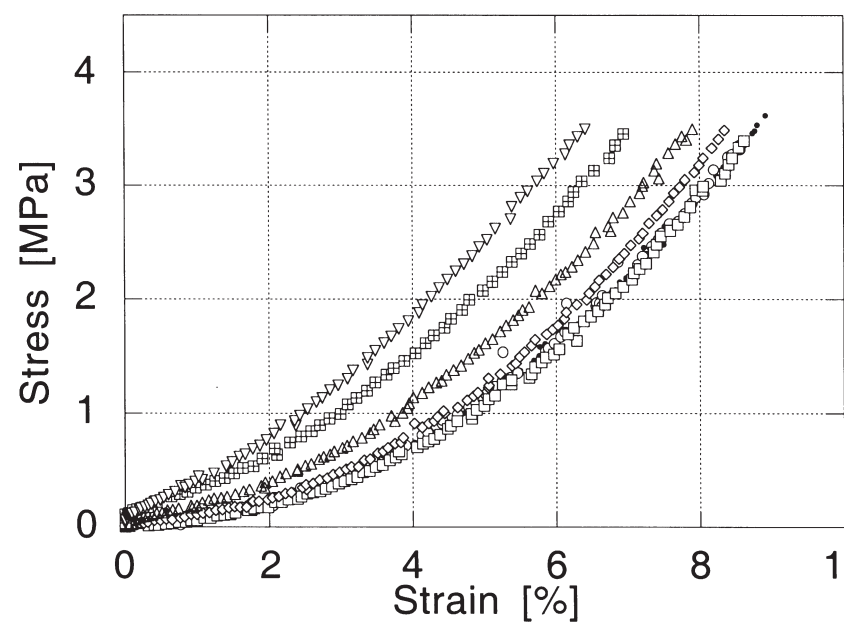

Fig. 3. Stress-strain curves for one ACL specimen at seven strain rates: •, $0.1 \% / \mathrm{s} ; \bigcirc, 1 \% / \mathrm{s} ; \square, 5 \% / \mathrm{s} ; \diamond, 10 \% / \mathrm{s} ; \triangle, 20 \% / \mathrm{s} ; \boxplus, 30 \% / \mathrm{s}$; $\nabla, 40 \% / \mathrm{s}$.

The "supplemental stress" clearly increased with the augmentation of the strain rate (Fig. 4). With a strain rate of $40 \% / \mathrm{s}$, the value of the "supplemental stress" could reach $70 \%$. It means that $70 \%$ of the stress in the ligament was due to the strain rate effect at a strain rate of $40 \% / \mathrm{s}$. This trend was found to be similar for all of the specimens tested (Table 3). The increase in "supplemental stress" with respect to the strain rate was statistically significant between all means reported in Table $3(p<0.0005)$.

\section{Discussion}

This study quantified the effect of strain rate on stresses in the ACL-bone complex. The hypothesis that the strain rate effect mainly acted in the toe region with

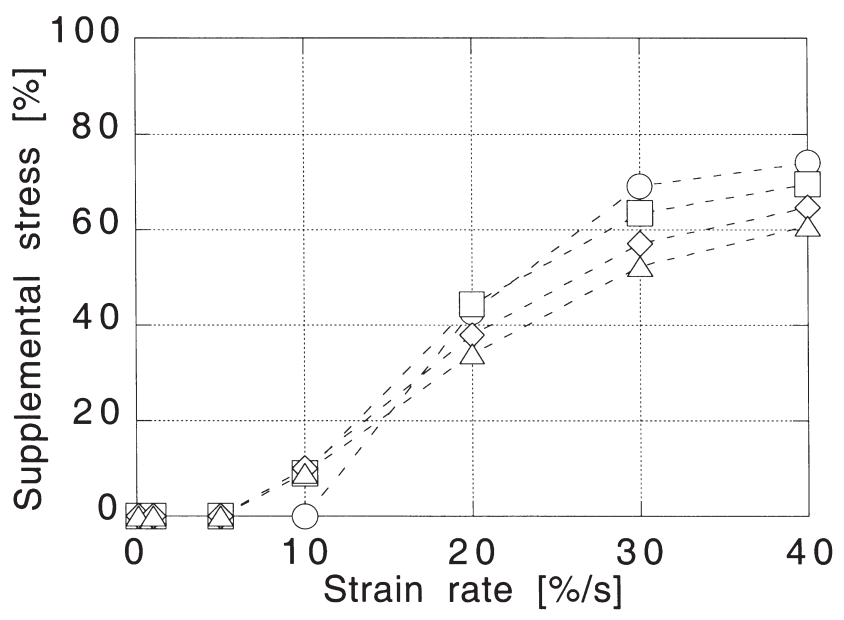

Fig. 4. "Supplemental stress" as a function of the strain rate at different strain rates for one ACL specimen: $\bigcirc, 1 \% ; \square, 2 \% ; \diamond, 3 \% ; \triangle, 4 \%$.

minimal influence on the linear part of the stress-strain curves was verified. This result allowed us to conclude that the use of the linear tangent moduli is, therefore, not adequate to characterize the strain rate effect in the ACL-bone complex. The "supplemental stress" was shown to be a synthetic and convenient variable to quantify the effect of the strain rate.

The traction tests were performed at room temperature. Using a similar protocol, it was found that the temperature parameter was of secondary importance in the mechanical results [17]. The traction tests on ACL can also be performed while immersed in a bath to control the temperature. If a bath is used however, the water content and the tonicity of the solution should be considered when examining viscoelastic properties $[18,19]$. In the present study, the specimens were constantly hydrated using a drip. The solution content certainly has less influence on the viscoelastic properties of drip-

Table 2

Mean values and standard deviations of the linear tangent moduli (11 specimens) for seven strain rates. No statistical difference was found between the mean values of the linear tangent moduli

\begin{tabular}{llllllll}
\hline & \multicolumn{2}{l}{ Strain rate $(\% / \mathrm{s})$} & & & \\
\cline { 2 - 8 } & 0.1 & 1 & 5 & 10 & 20 & 30 & 40 \\
\hline Linear tangent moduli $(\mathrm{MPa})$ & $69.2 \pm 13.1$ & $67.2 \pm 12.6$ & $72.4 \pm 13.5$ & $77.7 \pm 16.5$ & $76.6 \pm 14.2$ & $79.0 \pm 14.9$ & $83.5 \pm 16.2$ \\
\hline
\end{tabular}

Table 3

Mean values and standard deviations of the "supplemental stress" (11 specimens) for five strain rates. Statistical differences were found between all the mean values of the supplemental stress

\begin{tabular}{lllllll}
\hline & \multicolumn{3}{l}{ Strain rate $(\% / s)$} & & & \\
\cline { 2 - 6 } & 1 & 10 & 20 & 30 & 40 \\
\hline Supplemental stress (\%) (at 4\% strain) & $9.8 \pm 8.6$ & $26.4 \pm 9.2$ & $43.2 \pm 9.9$ & $58.9 \pm 8.2$ & $72.0 \pm 7.6$ \\
\hline
\end{tabular}


hydrated tissue than on those of tissue immersed in a bath. Moreover, to overcome this difficulty, the solution used for hydration was a physiological fluid and was identical for all the tests performed.

The cross-sectional area was assumed to be elliptic and the two principal diameters were measured with a caliper square. This measurement and, consequently, the values of the stress could be imprecise if the cross-sectional area of the specimens were quite different from an elliptical shape. The principal result of this study was the quantification of the strain rate effect. Precise crosssectional area measurement is then not essential as the "supplemental stress" variable is the ratio of two stress values.

This study showed that strain rate plays an important role in the mechanical behavior of the ACL-bone complex. The "supplemental stress" was found to increase considerably with the strain rate. The strain rate effect in the ACL has already been demonstrated in some previous studies but without specifically quantifying the effect of strain rate on the stress-strain curves. The measurement of one quantity only with respect to the strain rate, as the maximum load [20], furnished valuable information but did not describe the effect of the strain rate on the entire stress-strain curves. In the development of a constitutive law, the parameters of a model valid for the stress-strain curves obtained at one strain rate were expanded to take into account the results of tests made at different strain rates [9]. Results showed no monotonic increase of the parameters with respect to the strain rate, rendering the interpretation of the strain rate effect difficult. The present study uses a different approach. The definition of the "supplemental stress" enables the description of the effect of the strain rate on the entire stress-strain curves. The description of the entire stress-strain curves is important when comparing the mechanical behavior of the ACL with those of other tissues used as ACL grafts.

In this study, the shape of the stress-strain curves was shown not to be modified by the increase of the strain rate, except for the toe region which appeared at a lower magnitude of strain. This observation is similar to that reported in a previous study performed at lower strain rates $(<1 \% / \mathrm{s})$ [21]. The measurement of the linear tangent moduli did not show significant differences with respect to the strain rates and confirmed the observation that the strain rate effect acts mainly in the toe region. A direct consequence of this result is that the linear tangent moduli can not be used to quantify the effect of strain rate in a soft tissue as proposed for the patellar tendon specimens [22]. The quantification of the strain rate effect should use an independent variable, as the "supplemental stress" or should use a continuum mechanics approach as proposed in a recent paper [23].

We cannot then conclude, only with the measurement of the linear tangent moduli, that the mechanical proper- ties of a specimen is not sensitive to an increase of strain rate.

Some explanations for the toe region dependence on the strain rate can be based on the ultrastructure of the ACL. The shape of the stress-strain curve (toe region and linear region) has been observed to be due to the wavy pattern of the fibers comprising the ACL [24]. The toe region corresponds to the organization of the wavy pattern into an aligned fiber pattern, while the linear region corresponds to the strain of these aligned fibers. In recent studies, the strain rate sensitivity of the ligament has been related to the movement of water in the ligament [25]. It seems reasonable to believe that more water can move through the ligament during the early arrangement of the fibers as certainly more geometrical changes occur during this phase than during the extension of the aligned fibers. This would imply that a greater strain rate effect would be observed in the toe region.

It has been shown that the ACL function is to stabilize the knee, especially during anterior translation of the tibia with respect to the femur [26]. A dynamical stabilization of the knee also certainly occurs with the strain rate effect. If the motion of the tibia relative to the femur is slow, corresponding to a low strain rate for the ACL, it can be assumed that the muscles of the leg have enough time to stabilize the knee. The contribution of the ACL should be minimal in that case, which is well reflected by its less stiff stress-strain curve at low strain rates. If the motion of the tibia relative to the femur is fast, corresponding then to a high strain rate for the ACL, the muscles may not respond efficiently to stabilize the knee due to the time delay for their reaction. The contribution of the ACL to knee stability should, hence, increase. This could be achieved with a stiffer stressstrain curve and was what we observed in the traction tests at higher strain rates. It could be proposed then, that the mechanical response of the ACL is modulated by the speed of knee flexion.

In conclusion, the results of this study showed that the stress in the ACL-bone complex significantly increased with the strain rate and acted mainly in the toe region rendering the use of the linear tangent moduli not adequate to describe the strain rate effect. This study showed that the "supplemental stress" was a synthetic and convenient variable to quantify the effect of the strain rate on the entire stress-strain curves. This quantification is especially important when comparing the mechanical behavior of the ACL with those of other tissues used as ACL grafts.

\section{References}

[1] Fetto JE, Marshall JL. The natural history and diagnosis of anterior cruciate ligament insufficiently. Clin Orthop 1980;147:29-38. 
[2] McDaniel WJ, Dameron TB. Untreated ruptures of the anterior cruciate ligament. J Bone Joint Surg 1980;62A:696-704.

[3] Aglietti P, Buzzi R, D’Andria S, Zaccherotti G. Long-term study of anterior cruciate ligament reconstruction for chronic instability and using the central one-third patellar tendon and a lateral extraarticular tenodesis. Am J Sports Med 1992;20:38-45.

[4] Butler DL, Kay MD, Stouffer DC. Comparison of material properties in fascicle-bone units from human patellar tendon and knee ligaments. J Biomechanics 1986;19:425-32.

[5] Momersteeg TJA, Blankevoort L, Huiskes R, Kooloos JGM, Kauer JGM, Hendriks JCM. The effect of variable relative insertion orientation of human knee bone-ligament-bone complexes on the tensile stiffness. J Biomechanics 1995;28:745-52.

[6] Noyes FR, Delucas JL, Torvik PJ. Biomechanics of anterior cruciate ligament failure. An analysis of strain-rate sensitivity and mechanisms of failure in primates. J Bone Joint Surg 1974;56A:236-53.

[7] Race A, Amis AA. The mechanical properties of the two bundles of the human posterior cruciate ligament. J Biomechanics 1994;27:13-24.

[8] Butler DL, Guan Y, Kay M, Cummings J, Feder S, Levy M. Location-dependent variations in the material properties of the anterior cruciate ligament. J Biomechanics 1992;25:511-8.

[9] Danto MI, Woo SLY. The mechanical properties of skeletally mature rabbit anterior cruciate ligament and patellar tendon over a range of strain rates. J Orthop Res 1993;11:58-67.

[10] Woo SLY, Blomstrom GL. The tensile properties of the anterior cruciate ligament as a function of age. In: Jackson DW, Arnoczky SP, Frank CB, Woo SLY, Simon TN, editors. The anterior cruciate ligament: current and future concepts. New York: Raven Press, 1993:53-61.

[11] Pioletti DP. Viscoelastic properties of soft tissues: application to knee ligaments and tendons. Ph.D. thesis, EPF-Lausanne, 1997.

[12] Woo SLY, Orlando CA, Camp JF, Akeson WH. Effects of postmortem storage by freezing on ligament tensile behavior. J Biomechanics 1986;19:399-404.

[13] Pioletti DP, Rakotomanana L, Gillieron C, Leyvraz PF, Benvenuti, JF. Nonlinear viscoelasticity of the ACL: Experiments and theory. In: Middleton J, Jones NL, Pande GN, editors. Com- puter methods in biomechanics and biomedical engineering. Gordon \& Breach, Amsterdam, 1996:271-80.

[14] Yahia LH, Brunet J, Labelle S, Rivard CH. A scanning electron microscopic study of rabbit ligaments under strain. Matrix 1990;10:58-64.

[15] Hubbard RP, Chun KJ. Mechanical responses of tendons to repeated extensions and wait periods. J Biomech Engng 1988;110:11-19.

[16] Choi SC. Introductory applied statistics in science. PrenticeHall, 1978.

[17] Lam TC, Thomas CG, Shrive NG, Frank CB, Sabiston CP. The effects of temperature on the viscoelastic properties of the rabbit medial collateral ligament. J Biomech Engng 1990;112:147-52.

[18] Chimich D, Shrive N, Frank C, Marchuk L, Bray R. Water content alters viscoelastic behaviour of the normal adolescent rabbit medial collateral ligament. J Biomechanics 1992;25:831-7.

[19] Haut TL, Haut RC. The state of tissue hydratation determines the strain-rate-sensitive stiffness of human patellar tendon. J Biomechanics 1997;30:79-81.

[20] Kennedy JC, Hawkins RJ, Willis RB, Danylchuk KD. Tensions studies of human knee ligaments. J Bone Joint Surg 1976;58A:350-5.

[21] Haut RC, Little RW. Rheological properties of canine anterior cruciate ligaments. J Biomechanics 1969;2:289-98.

[22] Blevins FT, Hecker AT, Bigler GT, Boland AL, Hayes WC. The effects of donor age and strain rate on the biomechanical properties of bone-patellar tendon-bone allografts. Am J Sport Med 1994;22:328-33.

[23] Pioletti DP, Rakotomanana LR, Benvenuti JF, Leyvraz PF. Viscoelastic constitutive law in large deformations: application to knee ligaments and tendons. J Biomechanics 1998;31:753-7.

[24] Rigby B, Hirai N, Spikes J, Eryring H. The mechanical properties of rat tail tendon. J General Physiol 1959;43:123-32.

[25] Chen C-T, Vanderby R, Graft B. Interstitial fluid flow in ligaments and tendons: effects of fibril spacing and fluid properties. Transactions ASME Bioengineering Conference 1993;Bed24:399-402.

[26] Butler DL, Noyes FR, Grood ES. Ligamentous restraints to anterior-posterior drawer in the human knee. J Bone Joint Surg 1980;62A:259-70. 